\title{
Experiencia de implante percutáneo de la válvula aórtica en pacientes con estenosis aórtica severa descartados de cirugía convencional por alto riesgo quirúrgico en una comunidad rural
}

\author{
Experience of percutaneous aortic valve replacement in patients with \\ severe aortic stenosis discarded from conventional surgery due to high \\ surgical risk in a rural community
}

Federico Blanco', Rodrigo Blanco', Mauricio Bonet', Gustavo Iralde², Mariano Campeni ${ }^{3}$, Luis Blanco ${ }^{4}$, Guillermo González ${ }^{5}$, Pablo Guglienmone $^{6}$, Jorge Szarfer ${ }^{5}$, Alejandro García Escudero ${ }^{1}$

\section{RESUMEN}

Introducción. El reemplazo valvular aórtico quirúrgico es el tratamiento de elección en la estenosis aórtica severa sintomática. Ocasionalmente la edad avanzada y las comorbilidades imposibilitan la cirugía donde el reemplazo valvular percutáneo representa una alternativa válida.

Objetivo. Comunicar la experiencia del reemplazo percutáneo de la válvula aórtica en pacientes con estenosis aórtica severa y descartados de cirugía convencional por alto riesgo en una comunidad rural.

Material y métodos. Registro de 29 pacientes consecutivos con estenosis aórtica severa (área $\leq 1 \mathrm{~cm} 2$ ) sintomática y riesgo quirúrgico elevado sometidos a implante percutáneo entre el 01/12/2016 al 31/03/2018, utilizando una estrategia multidisciplinaria que involucró a diversos especialistas que conformaban el heart team. Se evaluaron las características basales de los pacientes, resultados del procedimiento, tipo de anestesia utilizada, sintomatología e internaciones en la evolución, y mortalidad al 01/04/2018.

Resultados. La edad fue de $80,65 \pm 6,5$ años, con $62 \%$ de mujeres. Comorbilidades: $21 \%$ diabéticos, 58,6\% enfermedad coronaria, 38\% disfunción ventricular izquierda, $24,1 \%$ EPOC, 58,5\% insuficiencia renal crónica, 6,8\% CRM y 52\% hipertensión pulmonar (tipo 2). Área valvular basal: $0,61 \pm 0,28 \mathrm{~cm} 2$. EuroSCORE: $16,9 \pm 8,5$. El 86,2\% presentaba disnea en clase funcional III y $13,8 \%$ presentaban disnea en clase funcional IV. Todos los procedimientos se realizaron con sedación y anestesia local y todos resultaron exitosos, con reducción del gradiente transvalvular pico de $78 \pm 16 \mathrm{mmHg}$ a $5 \pm 3 \mathrm{mmHg}(p<0,001)$ y ausencia de insuficiencia aórtica moderada o severa. El requerimiento de marcapasos definitivo fue $20,6 \%$. No hubo mortalidad en el procedimiento ni el seguimiento. Fl $89,6 \%$ evolucionó a clase funcional .

Conclusiones. El tratamiento de la estenosis aórtica severa en pacientes de alto riesgo quirúrgico mediante reemplazo valvular percutáneo sin anestesia general es una alternativa factible en pacientes de una comunidad rural. La selección adecuada, la sistematización del procedimiento y la interrelación multidisciplinaria aseguran su eficacia y seguridad.

Palabras claves: reemplazo percutáneo de válvula aórtica, estenosis aórtica severa, población rural, alto riesgo quirúrgico, sedación.

\begin{abstract}
Introduction. Surgical Aortic valve replacement (SAVR) is currently the treatment for the symptomatic aortic valve stenosis. In some patients were the surgical risk is very high due to age and comorbidities, the percutaneous valve replacement (TAVR) becomes a valid therapeutic alternative.

Objectives. To communicate the experience of TAVR performed in patients from a rural community with severe symptomatic aortic valve stenosis rejected from SAVR. Methods. Registry of 29 consecutive high surgical risk symptomatic aortic stenosis patients (area $\leq 1 \mathrm{~cm} 2$ ) undergoing to a percutaneous valve implantation between 01/12/2016 and 31/03/2018, indicated though a multi disciplinary decision of the site "Heart Team". The baseline characteristics of the patients, results of the procedure, type of anesthesia used, symptomatology and hospitalizations in the evolution and mortality at 01/April/2018 were evaluated.

Results. Mean age was $80,65 \pm 6,5$ years and $62 \%$ were females. Comorbidities: $21 \%$ were diabetics, $58,6 \%$ coronary heart disease, $38 \%$ left ventricular dysfunction, $24,1 \%$ chronic pulmonary obstructive disease, $58,5 \%$ chronic renal deficiency, $6,8 \%$ previous coronary bypass surgery y $52 \%$ Class 2 pulmonary hypertension. Pre treatment valvular area: $0,61 \pm 0,28 \mathrm{~cm} 2$. Euro SCORE: $16,9 \pm 8,5.86,2 \%$ of the population where in NYHA Class III dyspnea and $13,8 \%$ in Class IV. All the procedures were performed intravenous sedation and local anesthesia, 100\% of the replacements were successfully performed with a transvalvular peak gradient reduction from a pre procedure value of $78 \pm 16 \mathrm{mmHg}$ to $5 \pm 3 \mathrm{mmHg}$ after the TAVR $(p<0,001)$, y with no development of severe or moderate aortic valve regurgitation. Definitive pacemaker implantation was required in 20,6\% of the cases. There were no death immediately after procedure nor during follow up period with $89,6 \%$ of NYHA functional Class I.

Conclusions. TAVR elective treatment of severe aortic stenosis in a high surgical risk patients group with no general anesthesia at a rural community is a safe and feasible alternative. The adequate patient selection, together with the correct systematization of the procedure and the multidisciplinary actions ensures the efficiency and security for this procedure.
\end{abstract}

Key words: percutaneous aortic valve replacement, severe aortic stenosis, rural population, high risk surgery, sedation.

Revista Argentina de Cardioangiología Intervencionista 2019;10(2):58-62. https://doi.org/10.30567/RACI/201902/0058-0062

\footnotetext{
Servicio de Cardiología Intervencionista

Servicio de Electrofisiología

Servicio de Cirugía Cardiovascular

Servicio de Ecocardiografía

Servicio de Cardiología Clínica

Servicio de Anestesiología, Cardiovascular Chivilcoy, Provincia de Buenos Aires.

$\triangle$ Correspondencia: Dr. Federico Blanco. Miguel Calderón 79, B6620HLA Chivilcoy, Buenos Aires, Rep. Argentina. Tel: 02346-428352. federicoblanco@ymail. com
}

Los autores no declaran conflictos de intereses

Recibido: 23/01/2019|Aceptado:02/05/2019

\section{INTRODUCCIÓN}

La estenosis valvular aórtica es la enfermedad valvular más frecuente en el mundo occidental y tiene un pronóstico desfavorable con tratamiento médico. Debido a que la etiología más frecuente de la estenosis aórtica en los países occidentales es la degenerativa, la edad avanzada, con las comorbilidades presentes en este grupo etario, incrementan el riesgo quirúrgico y la morbilidad posoperatoria, por lo que existe una proporción considerable de pacientes cuyo riesgo quirúrgico impide la cirugía ${ }^{1-3}$, siendo el reemplazo valvular percutáneo el procedimiento de elección en esta po- 
TABLA 1. Características basales de la población $(n=29)$.

\begin{tabular}{|l|c|}
\hline Edad (años) & $80,6 \pm 6,5$ \\
\hline Sexo femenino \% (n) & $62(18)$ \\
\hline EuroSCORE logístico & $16,9 \pm 8,5$ \\
\hline Disnea NYHA III \% (n) & $86,2(25)$ \\
\hline Disnea NYHA IV \% (n) & $13,8(4)$ \\
\hline Enfermedad coronaria \% (n) & $58,5(17)$ \\
\hline Cirugía cardíaca previa \% (n) & $6,8(2)$ \\
\hline Diabetes \% (n) & $20,6(6)$ \\
\hline Enfermedad vascular periférica \% (n) & $79,3(23)$ \\
\hline EPOC \% (n) & $24,1(7)$ \\
\hline Disfunción renal \% ( $n$ ) & $58,5(17)$ \\
\hline Hipertensión pulmonar \% (n) & $52(15)$ \\
\hline Antecedente de radiación torácica \% (n) & $6,8(2)$ \\
\hline Antecedente de valvuloplastia aórtica & $6,8(2)$ \\
\hline Fibrilación auricular \% (n) & $6,8(2)$ \\
\hline Fracción de eyección & $51,5 \pm 16 \%$ \\
\hline Fracción de eyección <50\% & $37,9(11)$ \\
\hline Fracción de eyección <30\% & $10,3(3)$ \\
\hline Área valvular aórtica (cm²) & $0,61 \pm 0,28$ \\
\hline Gradiente medio (mmHg) & $48,2 \pm 16$ \\
\hline Gradiente máximo (mmHg) & $78 \pm 16$ \\
\hline
\end{tabular}

blación ${ }^{4,5}$. Las guías terapéuticas actuales indican el uso de este procedimentos a aquellos pacientes con estenosis aórtica severa sintomática considerados inoperables por el equipo multidisciplinario (indicación I, nivel de evidencia B), o a los que que, siendo operables, tuvieran riesgo quirúrgico elevado (indicación IIa, nivel de evidencia B) ${ }^{6}$. El objetivo de este estudio es presentar la experiencia de un grupo de trabajo consolidado respecto del implante percutáneo de válvula aórtica realizadas en una institución localizada en una ciudad rural, que recibe pacientes de la misma ciudad o de poblaciones rurales vecinas, luego de haber alcanzado una sistematización de la técnica, con un seguimiento hasta la fecha de corte del estudio.

\section{MATERIAL Y MÉTODOS}

\section{Diseño del estudio}

Luego de la experiencia inicial realizada entre 2013 y 2016 y obtener, cada uno de los 4 cardiólogos intervencionistas del Servicio de Hemodinamia, la certificación habilitante para realizar en forma independiente el procedimiento, se inició este registro prospectivo, evaluados por un equipo multidisciplinario de cardiólogos clínicos, ecocardiografistas, electrofisiólogos, cardiólogos intervencionistas, cirujanos cardiovasculares y anestesiólogos (el heart team). Se incluyeron 29 pacientes en forma consecutiva con estenosis aórtica severa sintomática (área $<1 \mathrm{~cm}^{2}$ ), de alto riesgo quirúrgico, descartados de tratamiento quirúrgico convencional, sometidos a un implante percutáneo de válvula aórtica entre el 01/12/2016 y el 31/03/2018. A los mismos se les realizaron las siguientes exploraciones antes del procedimiento: examen prequirúrgico de laboratorio, ecocardiograma transtorácico y/o transesofágico (si el estudio transtorácico no era concluyente), espirometría, eco doppler arterial de miembros inferiores y de los vasos del cuello, coronariografía y angiotomografía de aorta torácica, abdominal y de ejes ilíaco-femorales. El riesgo quirúrgico se calculó con el EuroSCORE. La enfermedad coronaria concomitante se trató por vía endovascular antes del reemplazo valvular. Se definió éxito del procedimiento al implante correcto de la prótesis con caída significativa del gradiente transavalvular en
TABLA 2. Procedimiento $(n=29)$.

\begin{tabular}{|l|c|}
\hline Anestesia: técnica de sedación y anestesia local \% (n) & $100(29)$ \\
\hline Mortalidad \% (n) & 0 \\
\hline Taponamiento cardíaco \% (n) & $3,4 \%(1)$ \\
\hline Accidente cerebrovascular \% (n) & $3,4 \%(1)$ \\
\hline Complicaciones vasculares \% (n) & $3(2-5)$ \\
\hline Internación (días) mediana (IIC) & $5 \pm 3$ \\
\hline Gradiente máximo post-TAVI (mmHg) & \\
\hline Insuficiencia aórtica & $44,8(13)$ \\
\hline 0\% (n) & $51,7(15)$ \\
\hline I \% (n) & $3,4(1)$ \\
\hline II \% (n) & 0 \\
\hline III-IV \% (n) & $20,7(6)$ \\
\hline Implante de marcapasos definitivo \% (n) & $72,4(21)$ \\
\hline Requerimiento de valvuloplastia pre implante valvular \% (n) & $24,1(7)$ \\
\hline Requerimiento de valvuloplastia post implante valvular \% (n) & $20,7(6)$ \\
\hline $\begin{array}{l}\text { Sin requerimiento de valvuloplastia en el implante valvular } \\
\%(n)\end{array}$ & $44,8(13)$ \\
\hline Tamaño de prótesis valvular & $44,8(13)$ \\
\hline$N^{\circ} 26 \%$ (n) & $10,3(3)$ \\
\hline$N^{\circ} 29 \%$ (n) & \\
\hline$N^{\circ} 31 \%$ (n) & \\
\hline
\end{tabular}

ausencia de regurgitación aórtica de grado moderado o severo (evaluada por angiografía y ecocardiograma Doppler), sin mortalidad al alta hospitalaria. El punto final del estudio fue muerte, stroke inhabilitante, y/o insuficiencia aórtica severa a los 30 días.

\section{Procedimiento}

El implante de las válvulas se llevó a cabo en el laboratorio de hemodinamia mediante anestesia local y sedación profunda. Una vez sedado el paciente, en forma simultánea el electrofisiólogo implanta por vía yugular derecha el catéter de marcapasos (catéter de fijación activa en todos los casos) y el cirujano cardiovascular realiza el acceso vascular mediante disección de la arteria femoral. Luego, el cardiólogo intervencionista, por punción coloca un introductor en la femoral contralateral, para avanzar un catéter pig-tail hasta posicionarlo en el seno no coronariano para medir gradiente de presiones y el control angiográfico. Por la arteria femoral reparada por disección se coloca un introductor 18 Fr. A través del cual se progresa un catéter AL2 y con cuerda recta se traspone el plano valvular. Se progresa un catéter pig-tail al ventrículo izquierdo y se registra el gradiente transvalvular aórtico por registro simultáneo de presión aórtica y ventricular. Luego se posiciona en el ventrículo izquierdo una cuerda Amplatz ${ }^{\circ}$, Confida o Safari ${ }^{\circ}$. A continuación, en los casos con severa calcificación, se realiza valvuloplastia aórtica con un balón de diámetro similar al del anillo valvular mientras se estimula con marcapasos a 180 por minuto hasta lograr el descenso de la presión sistémica menor a $50 \mathrm{mmHg}$. Posteriormente, se avanza la válvula por el introductor 18 Fr. hasta posicionarla a nivel del anillo valvular. Se libera la misma, requiriendo en ocasiones la estimulación con marcapasos. Se evalúa nuevamente el gradiente transvalvular, la altura del implante y la presencia de insuficiencia valvular. Si se constata estenosis o insuficiencia valvular significativa, se dilata la válvula protésica con un balón de mayor diámetro. Finalmente se procede a la síntesis quirúrgica de la arteria femoral.

\section{Cuidados luego el procedimiento}

Luego del procedimiento, los pacientes permanecieron monitorizados en Terapia Intensiva durante por lo menos 36 
TABLA 3. Seguimiento de la población.

\begin{tabular}{|l|c|}
\hline Seguimiento (días) mediana (IIC) & $161(77-300)$ \\
\hline NYHA I \% (n) & $89,1(26)$ \\
\hline Área valvular post-TAVI (cm²) & $1,78 \pm 2$ \\
\hline Gradiente medio post-TAVI (mmHg) & $10,3 \pm 9$ \\
\hline Mortalidad \% (n) & 0 \\
\hline Internaciones \% ( $\mathrm{n})$ & $10,3(3)$ \\
\hline
\end{tabular}

horas. A los que no presentaban trastornos del ritmo se les retiraba el marcapasos transitorio. Si durante ese período se registraba algún episodio de bloqueo auriculoventricular, se implantaba el marcapasos definitivo.

\section{Seguimiento}

El seguimiento luego del alta sanatorial fue realizado a los 15 y 30 días y a los 6 meses en el consultorio, donde se realizaba examen físico completo y se constataba evolución, síntomas y clase funcional (NYHA). Ecocardiograma control a los 30 días, 6 y 12 meses. El seguimiento en la evolución fue coordinado con el cardiólogo de cabecera de la localidad de origen del paciente. Más allá de las visitas pautadas, se realizó seguimiento telefónico para determinar evolución de los síntomas y posibles intercurrencias que pudieran surgir. Con el fin de contar con los datos para la presente publicación, los pacientes fueron contactados telefónicamente durante la primera semana del mes de abril del 2018, registrando sobrevida, internaciones, intercurrencias y su clase funcional.

\section{Análisis estadístico}

Las variables categóricas se expresan como porcentajes. Las variables continuas de distribución normal se presentan como media y desvío estándar (DE) y, cuando son de distribución no gaussiana, como mediana e intervalo intercuartilo (IIC). Las variables categóricas fueron comparadas con tablas de contingencia, con test de Chi cuadrado con corrección de Yates o Fisher según correspondiera. La comparación de las variables continuas se realizó mediante el test de T o con tests no paramétricos (Wilcoxon y Kruskal-Wallis) según su distribución. El análisis fue realizado con el programa Statistix 7.0 y se consideró significativa una $\mathrm{p}<0,05$.

\section{RESULTADOS}

Se incluyeron en el estudio los 29 pacientes consecutivos a los que se les realizó el implante percutáneo de válvula aortica entre el 01/12/2016 y el 31/03/2018 en un sanatorio de una comunidad rural. La edad media de la población fue de $80,6 \pm 6,5$ años. Todos los pacientes presentaban estenosis aórtica severa sintomática. El área valvular media me-

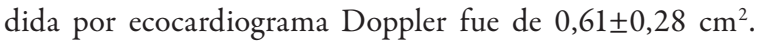
El $86,2 \%$ de los pacientes se encontraban en clase funcional III y $13,8 \%$ en clase funcional IV. El EuroSCORE fue de 16,9 $\pm 8,5$ (Tabla 1). El 38\% de los pacientes eran varones, el $21 \%$ diabéticos, el $58,6 \%$ tenía antecedentes de enfermedad coronaria, el $52 \%$ presentaba distintos grados de hipertensión pulmonar (tipo 2), el 37,9\% deterioro de la función ventricular izquierda, el 24,1\% EPOC, el 58,5\% insuficiencia renal crónica y el 6,8\% cirugía de revascularización previa. Dos pacientes tenían antecedentes de radioterapia torácica por neoplasia y presentaban tórax hostil. Dos pacientes presentaban el antecedente de valvuloplastia aórtica previa por insuficiencia cardíaca refractaria al tratamiento médico (Tabla 1). La totalidad de los procedimientos se reali- zaron con sedación profunda y anestesia local. Un paciente requirió asistencia respiratoria mecánica por 48 horas luego del procedimiento debido a depresión respiratoria. En todos los procedimientos se realizó disección de la arteria femoral y se colocó un catéter de fijación activa para el marcapasos transitorio. En 28 casos $(96,6 \%)$ se implantaron válvulas autoexpandibles (CoreValve y CoreValve Evolut R, Medtronic, USA), mientras que en el caso restante se implantó una válvula balón expandible (Edwards SAPIEN-XT, Edwards Lifesciences, USA). Todos los procedimientos resultaron exitosos traduciéndose en una reducción pronunciada del gradiente pico transvalvular aórtico $(78 \pm 16$ vs. $5 \pm 3 \mathrm{mmHg}$; $\mathrm{p}<0,001)$, sin desarrollo posprocedimiento de regurgitación aórtica de grado moderado o severo. Respecto del tamaño de las válvulas implantadas, en 13 ocasiones se implantó una válvula $\mathrm{N}^{\circ} 26$, en otras 13 ocasiones la válvula implantada fue $\mathrm{N}^{\circ} 29$ y en las 3 restantes se implantó una válvula $\mathrm{N}^{\circ}$ 31. Durante el implante, en 21 casos se realizó valvuloplastia previa al implante protésico, 7 casos requirieron valvuloplastia posterior al implante valvular y 6 casos no requirieron valvuloplastia en ningún momento (Tabla 2).

Los trastornos de conducción representaron una intercurrencia frecuente. El electrocardiograma basal mostró ritmo sinusal en 27 pacientes. Los 2 restantes registraban fibrilación auricular (6,8\%). Los trastornos de conducción basales fueron bloqueo completo de rama derecha (BCRD) en 1 paciente, BCRD + hemibloqueo anterior izquierdo (HBAI) en 1 paciente y bloqueo completo de rama izquierda (BCRI) en 3 pacientes. Luego del procedimiento, 13 pacientes presentaron nuevo BCRI (50\%) y 6 pacientes requirieron el implante de un marcapasos definitivo (20,68\%), 5 por bloqueo auriculoventricular (BAV) completo intraprocedimiento y 1 por BCRI con BAV de primer grado con HV prolongado. De estos 6 pacientes, 2 presentaban alteraciones de la conducción en el electrocardiograma basal (1 BCRD + HBAI y 1 BCRI), siendo la tasa de requerimiento de implante de marcapasos definitivo en pacientes con trastornos de conducción basal del 40\% ( $R R=2,4$; IC95\%: 0,59-9,7; $p=0,24)$. Un paciente requirió la realización de drenaje pericárdico por signos de taponamiento cardíaco a las 24 horas de realizado el procedimiento. La duración de la internación fue de 3.58 \pm 1,52 días (Tabla 2). No se registraron muertes intraprocedimiento, durante la internación, ni en el seguimiento hasta el 1 de abril de 2018 (mediana de seguimiento: 161 días; IIC: 77-300 días). Un paciente no pudo ser localizado telefónicamente la primera semana de abril de 2018 (fecha de corte del estudio), pero se encontraba asintomático las visitas presenciales previas. Tres pacientes necesitaron internarse en el seguimiento, 2 por disnea y el restante por pseudoaneurisma en el sitio donde se colocó el introductor arterial (arteria contralateral a la arteria en que se le realizó disección). Se observó en el seguimiento mejoría de la clase funcional en la totalidad de los pacientes, con una evolución a clase funcional I en el 89,6\% (Tabla 3).

\section{DISCUSIÓN}

En los últimos años, el implante percutáneo de válvula aórtica se ha consolidado como una alternativa eficaz y segura al tratamiento quirúrgico para pacientes con estenosis aórtica severa inoperables o con alto riesgo quirúrgico ${ }^{1,2}$. En el presente estudio mostramos nuestra experiencia respecto del implante valvular percutáneo sin anestesia general en 
pacientes con esta patología, llevada a cabo en una comunidad rural, en pobladores de la misma y de ciudades vecinas con similares características demográficas. La necesidad de brindar una adecuada atención médica plantea un dilema a las instituciones de entornos rurales, que pueden presumir mayores desafíos, con exigencia de mejores resultados basados en una histórica percepción de rendimiento inferior en procedimientos que requieran mayor tecnología en estas áreas ${ }^{7,8}$. En nuestro caso, los resultados fueron definitivamente comparables, si no mejores, que los de otros registros nacionales ${ }^{9}$. La ausencia de mortalidad en los 29 casos incluidos en este estudio, el éxito en la totalidad de los procedimientos y la mejoría significativa de la clase funcional de los pacientes avalan esta afirmación. Dichos resultados pudieron ser logrados debido a la experiencia en este tipo de implante de cualquiera de los 4 cardiólogos intervencionistas que conforman el Servicio de Hemodinamia, que intervinieron indistintamente como primero o segundo operador, y a la sistematización del procedimiento con el resto del equipo médico, conformado por el cirujano cardiovascular, el electrofisiólogo, el ecocardiografista, el anestesiólogo y el equipo paramédico. Además de tener una estadía hospitalaria corta (internación promedio 3.58 $\pm 1,52$ días), el análisis costo/beneficio de realizar este tipo de procedimientos en la ciudad del paciente o en ciudades vecinas también podría considerarse como superior, debido a que permite mantener al paciente en su ámbito en contacto permanente con el médico de cabecera encargado del seguimiento, evitando además gastos en traslado y mayores costos por alojamiento y alimentación de los acompañantes en ciudades de mayor población ${ }^{8}$. La corta estadía hospitalaria, además de la sistematización del procedimiento y del seguimiento del paciente, tiene estrecha relación con la realización del implante mediante anestesia local y sedación profunda en la totalidad de los casos, evitando la intubación orotraqueal ${ }^{10-12}$.

A pesar de estos resultados alentadores, es importante poner especial énfasis en las intercurrencias agudas derivadas del procedimiento, a expensas de los trastornos de conducción y de taponamiento cardíaco en un caso. Respecto de los trastornos de conducción luego del procedimiento, 13 pacientes presentaron nuevo BCRI (50\%) y 6 pacientes requirieron el implante de un marcapasos definitivo (20,68\%). El motivo del implante fue BAV completo intraprocedimiento en 5 pacientes, y BCRI con BAV de primer grado con HV prolongado en el estudio electrofisiológico en el restante. Este dato coincide con trabajos recientemente publicados ${ }^{13-15}$. Si bien la tendencia del uso del marcapasos ha ido disminuyendo, nuestra tasa sobre su empleo coincide con la de otros registros contemporáneos ${ }^{16-18}$. A su vez, esta tasa es mayor cuando el tipo de válvula utilizada es la más usada en este registro ${ }^{16-19}$. En cuanto a la prevención del taponamiento cardíaco, intercurrencia observada en un caso de nuestra serie, con tasa coincidente con otros registros ${ }^{20,21}$, consideramos un factor muy importante la utilización de cuerdas preformadas (Safari ${ }^{\circ}$, Confida o similares) o preformarlas en forma adecuada, así como la visión continua del borde distal de esta mediante control fluoroscópico para detectar rápidamente desplazamientos que conlleven riesgo de perforación. En este sentido también es muy importante el uso de catéteres de marcapasos endocavitarios de fijación activa y su correcto y cuidadoso posicionamiento mediante un operador con adecuada experiencia, en nuestro caso el electrofisiólogo.
Con respecto al seguimiento, este se cerró transitoriamente durante la primera semana de abril de 2018 , con una mediana de 161 (IIC: 77-300) días, a fin de realizar una evaluación preliminar para ser publicada en esta oportunidad. Solo un paciente y sus familiares no pudieron ser localizados. En este período de tiempo hemos objetivado mejoría significativa en la clase funcional de los pacientes, sin óbitos hasta dicha fecha. La información actualmente disponible sobre el seguimiento a largo plazo de pacientes a quienes se les realizó implante percutáneo de válvula aórtica es muy escasa. Las series publicadas actualmente se pueden analizar según el tipo de válvula empleada, Edwards SAPIEN y CoreValve, dado que estas son las válvulas inicialmente implantadas y, por lo tanto, las que acumulan los seguimientos más prolongados ${ }^{22-24}$. Se puede asumir que la mortalidad total a los 5 años oscila entre el 50-70\% ${ }^{24,25}$. Según los datos publicados, la mayor tasa de mortalidad ocurre en el primer año de seguimiento, explicada por la mayor tasa de mortalidad en el primer mes a consecuencia de complicaciones derivadas del procedimiento $^{21,25}$. Tras el alta hospitalaria, la principal causa de muerte es la no cardíaca, por la elevada tasa de comorbilidades que presenta esta población. Las infecciones complicando afecciones previas y la insuficiencia respiratoria son causas de muerte muy prevalentes, seguidas por el desarrollo de otras enfermedades relacionadas con el envejecimiento, como la insuficiencia cardiaca, la insuficiencia renal, el fallo multiorgánico y el cán$\operatorname{cer}^{21,24-26}$. Al no haber presentado en nuestra serie mortalidad asociada al procedimiento, cobra importancia el seguimiento alejado de esta población con el fin de cuantificar la sobrevida y determinar las causales de muerte.

Tal como fue observado en este registro, el implante percutáneo de la válvula aórtica, produce un beneficio en la sobrevida y en la calidad de vida de los pacientes, mejorando la evolución natural de esta patología ${ }^{1,2,20,21}$. Este beneficio no solo se da por un incremento del acceso al tratamiento de pacientes portadores de esta patología con imposibilidad de la resolución quirúrgica, sino también por mejoría de los resultados en pacientes sometidos a reemplazo convencional, debido a una mejor selección del paciente y a una derivación más precoz respecto del curso de la enfermedad ${ }^{27}$. A pesar de un peor perfil clínico, el implante percutáneo de válvula aórtica presenta una menor tasa de mortalidad comparada con la cirugía convencional en pacientes de 75 años o mayores ${ }^{27}$. Nuestros resultados podrían tener implicancia para el tratamiento de esta patología en nuestro país.

\section{CONCLUSIONES}

El tratamiento de la estenosis aórtica severa mediante reemplazo valvular percutáneo sin anestesia general para pacientes de alto riesgo quirúrgico descartados de cirugía convencional es una alternativa factible en aquellos pertenecientes a una comunidad rural. La selección adecuada de nuestros pacientes, la sistematización de la técnica del procedimiento y la interrelación multidisciplinaria incrementan su eficacia y seguridad.

\section{AGRADECIMIENTO}

Los autores le agradecen a nuestras coordinadoras administrativas Vanesa Schmidt, Carla Poltroni y Mónica Ruckauf y a nuestros técnicos Pablo Oliver, Alan Burcez y Alejandro Vera, sin los cuales no hubiésemos podido realizar los casos y por ende, presentar este trabajo. 


\section{BIBLIOGRAFÍA}

1. Leon M, Smith C, MackM, et al. PARTNER Trial Investigators. Transcatheter aortic-valve implantation for aortic stenosis in patients who cannot undergo surgery. N Engl J Med 2010;363:1597-607.

2. Agatiello C, Candiello A, Sztejfman M, y cols. Consenso de implante percutáneo del Colegio Argentino de Cardioangiólogos Intervencionistas 2016. Revista Argentina de Cardioangiología Intervencionista 2016;7(4):194-210. https://DOl.org/10.30567/ RACI/201604/0194-0210

3. Adams D, Popma J, Reardon $M$, et al. Core Valve Clinical Investigators. Transcatheter aortic-valve replacement with a selfexpanding prosthesis. N Engl J Med 2014;370:1790-8.

4. Makkar RR, Fontana GP, Jilaihawi H, et al. for the PARTNER Trial Investigators. Transcatheter aortic-valve replacement for inoperable severe aortic stenosis. N Engl J Med 2012;366:1696-704.

5. Smith CR, Leon MB, Mack MJ, et al. PARTNER Trial Investigators. Transcatheterversus surgical aortic-valvereplacementin high-riskpatients. NEngl J Med 2011:364:2187-98.

6. Vahanian A, Alfieri $O$, Andreotti F, et al. Guidelines on the management of valvular heart disease (version 2012). Eur Heart J 2012;33:2451-96.

7. Stys T; Stys A; Raizada A, et al. Rural United States experience of incorporation of a technologically advanced and procedurally complex cardiovascular program- The Sanford trans-catheter aortic valve replacement experience. South Dakota Medicine J 2016;69:351-8.

8. Torzewski J, Zimmermann O, Paula J, et al. In-hospital results of transcatheter aortic valve implantation (TAVI) in a district hospital--an approach to treat TAVI patients in rural areas. Int J Cardiol 2013 Oct 12;168(5):4845-6.

9. Cura F, Candiello A, Londero H, y cols. Reemplazo percutáneo de la válvula aórtica en pacientes con estenosis aórtica grave y riesgo quirúrgico elevado. Rev Argent Cardiol 2011; 79:314-21.

10. Hyman MC, Vemulapalli S, Szeto W, et al. Conscious Sedation Versus General Anesthesia for TranscatheterAortic ValveReplacement:Insightsfrom the National Cardiovascular Data Registry Society of Thoracic Surgeons/ American College of Cardiology Transcatheter Valve Therapy Registry. Circulation 2017:136(22):2132-40.

11. Caponi G, Fava C, Fraguas H, y cols. Evolución de la experiencia con reemplazo de válvula aórtica (TAVR) en un centro de Argentina. Revista Argentina de Cardioangiología Intervencionista 2016;7(4):211-16. https://DOI. org/10.30567/RACI/201604/0211-0216

12. Husser O'Fujita B, Hengstenberg C, et al. Conscious Sedation Versus General Anesthesia in Transcatheter Aortic Valve Replacement. The German Aortic Valve Registry. J Am Coll Cardiol Intv 2018;11:567-78.

13. Maan A, RefaatMM, Heist EK, et al. Incidence and Predictors of Pacemaker Implantation in Patients Undergoing Transcatheter Aortic Valve Replacement. Pacing Clin Electrophysiol 2015;38:878-86.
14. Kostopoulou A, Karyofillis P, Livanis E, et al. Permanent pacing after transcatheteraortic valve implantation of a CoreValve prosthesis as determined by electrocardiographic and electrophysiological predictors: a single-centre experience. Europace 2016;18(1):131-7

15. López-Aguilera J, Segura Saint-Gerons JM, Mazuelos Bellido F, et al. Effect of newonset left bundle branch block after transcatheter aortic valve implantation (Corevalve) on mortality, frequency of re-hospitalization, and need for pacemaker. Am J Cardiol 2016;118:1380-5.

16. Di Mario C, Eltchaninoff H, Moat N, et al. Valve Treatment Sentinel Registry (TCVT) Investigators of the EURObservational Research Programme (EORP) of the European Society of Cardiology. The 2011-12 pilot European Sentinel Registry of Transcatheter Aortic Valve Implantation: in-hospital results in 4,571 patients. Eurolntervention 2013;8:1362-71.

17. de Brito FS Jr, Carvalho LA, Sarmento-Leite R, et al. Brazilian TAVI Registry Investigators. Outcomes and predictors of mortality after transcatheter aortic valve implantation: results of the Brazilian registry. Catheter Cardiovasc Interv 2015;85:E153-E162

18. Meredith IT, Walton A, Walters DL, et al. Mid-term outcomes in patients following transcatheter aortic valve implantation in the CoreValve Australia and New Zealand Study. Heart Lung Circ 2015;24:281-90.

19. Auffret $V$, Puri $R$, Urena M, et al. Conduction Disturbances After Transcatheter Aortic Valve Replacement Current Status and Future Perspectives. Circulation 2017;136:1049-69.

20. Kalra S, Firoozi S, Yeh J, et al. Initial Experience of a Second-Generation Self-Expanding Transcatheter Aortic Valve: The UK \& Ireland Evolut R Implanters' Registry. JACC Cardiovasc Interv 2017;10(3):276-82.

21. Ussia G, Barbanti M, Petronio A, et al. Transcatheter aortic valve implantation: 3-year outcomes of self-expanding CoreValve prosthesis. Eur Heart J 2012;33:969-76.

22. Toggweiler S, Humphries KH, LeeM, et al. 5-year outcome after transcatheter aortic valve implantation. J Am Coll Cardiol 2013; 61:413-19.

23. Unbehaun A, Pasic M, Drews T, et al. Transapical aortic valve implantation: predictors of survival up to 5 years in 730 patients. An update. Eur $\mathrm{J}$ Cardiothorac Surg 2015:47:281-90

24. Kapadia S, Tuzcu E, Makkar R, et al. Long-term outcomes of inoperable patients with aortic stenosis randomized to transcatheter aortic valve replacement or standard therapy. Circulation 2014;130:1483-92.

25. Barbanti M, Petronio AS, Ettori F, et al. 5-year outcomes after transcatheter aortic valve implantation with CoreValve prosthesis. JACC CardiovasC Interv 2015:8:1084-91

26. Codner P, Orvin K, Assali A, et al. Long-term outcomes for patients with severe symptomatic aortic stenosis treated with transcatheter aortic valve implantation. Am J Cardiol 2015:116:1391-98.

27. Nguyen $V$, Michel M, EltchaninoffH, et al. Implementation of Transcatheter Aortic Valve Replacement in France. J Am Coll Cardiol 2018;71:1614-27. 